\title{
Rupturas da Aliança Terapêutica: um estudo de caso malsucedido em psicoterapia psicanalítica com paciente borderline
}

\section{Alliance Rupture: an unsuccessful case of psychoanalytic psychotherapy with a borderline patient}

\section{Rupturas de la Alianza Terapéutica: un estudio de caso interrumpido en psicoterapia psicoanalítica con un paciente limítrofe}

\author{
Patricia Dotta ${ }^{1}$, ORCID 0000-0003-3089-2348 \\ Luan Paris Feijó ${ }^{2}$, ORCID 0000-0002-7587-3987 \\ Fernanda Barcellos Serralta ${ }^{3}$, ORCID 0000-0003-4602-6495 \\ ${ }^{123}$ Universidade do Vale do Rio dos Sinos. Brasil
}

Resumo: Esse trabalho tem como objetivo compreender os processos de ruptura da Aliança Terapêutica (AT) de um caso de psicoterapia psicanalítica (PP) interrompida, realizada com paciente com Transtorno de Personalidade Borderline (TPB). Trata-se de estudo de caso sistemático, que contempla 15 sessões de PP, com um paciente com queixas impulsividade e dificuldades de relacionamento e sua terapeuta. As sessões foram gravadas em vídeo e transcritas. A identificação das rupturas na AT foi feita pelo Rupture Resolution Rating System. Foram encontradas 100 rupturas da AT, destas 69\% são rupturas de evitação e $31 \%$ de confrontação. Foram verificadas 30 contribuições da terapeuta para as rupturas acontecerem. As rupturas de evitação são mais sutis e de difícil identificação, apresentandose em maior frequência do que as de confrontação. Diante de pacientes com TPB, os terapeutas devem desenvolver habilidades para fazer intervenções com foco sobre a AT. Destaca-se a necessidade de outros estudos que busquem replicar a pesquisa em outros casos, de sucesso e insucesso terapêutico.

Palavras-chave: rupturas; aliança terapêutica; psicoterapia; investigação de processos; transtorno de personalidade borderline

Abstract: This paper aims to understand the processes of rupture of the Therapeutic Alliance (TA) of a case of interrupted psychoanalytic psychotherapy (PP) with a patient with Borderline Personality Disorder (BPD). This is a systematic case study that comprises 15 sessions of PP, one patient with complaints of impulsiveness and difficulties in interpersonal relationships, and his female therapist. The sessions were videotaped and transcribed. The identification of ruptures was made by the Rupture Resolution Rating System (3R's). There were 100 ruptures of AT, of these $69 \%$ were withdrawal ruptures and $31 \%$ of confrontation. We found 30 contributions from therapist to ruptures. The withdrawal ruptures are more subtle and difficult to identify, occurring more frequently than those of confrontation in the treatment. In the case of patients with BPD, therapists should develop skills to make interventions focused on TA. The need for other studies that seek to replicate the research in other cases of success and therapeutic failure is highlighted.

Keywords: ruptures; therapeutic alliance; psychotherapy; process research; borderline personality disorder 
Resumen: Este trabajo tiene como objetivo comprender los procesos de ruptura de la Alianza Terapéutica (AT) de un caso de psicoterapia psicoanalítica (PP) interrumpida realizada con un paciente con Trastorno de Personalidad Borderline (TPB). Se trata de un estudio de caso sistemático, el caso contempla 15 sesiones de PP, un paciente con quejas de impulsividad y dificultades de relación y su terapeuta. Las sesiones fueron grabadas en vídeo y transcritas. La identificación de las rupturas en la AT fue hecha por el Rupture Resolution Rating System. Se encontraron 100 rupturas de la AT, de estas $69 \%$ son rupturas de evitación y $31 \%$ de confrontación. Han sido verificadas 30 contribuciones del terapeuta para las rupturas. Las rupturas de evitación son más sutiles y de difícil identificación, presentándose en mayor frecuencia que las de confrontación en el tratamiento. Ante los pacientes con TPB los terapeutas deben desarrollar habilidades para hacer intervenciones con foco sobre la AT. Se destaca la necesidad de otros estudios que busquen replicar la investigación en otros casos de éxito y fracaso terapéutico.

Palabras clave: rupturas; alianza terapéutica; psicoterapia; investigación de procesos; Trastorno de Personalidad Limítrofe

Recebido: 10/06/2019

Aceito: $16 / 09 / 2020$

\section{Como citar:}

Dotta, P., Feijó, L.P., \& Serralta, F.B. (2020). Rupturas da Aliança Terapêutica: um estudo de caso malsucedido em psicoterapia psicanalítica com paciente borderline. Ciencias Psicológicas, 14(2), e-2321. doi: https://doi.org/10.22235/cp.v14i2.2321

Correspondência: Patricia Dotta; Universidade do Vale do Rio dos Sinos. E-mail: patricia.dotta5@yahoo.com.br. Luan Paris Feijó; e-mail: lparisf@gmail.com. Fernanda Barcellos Serralta; e-mail: fernandaserralta@gmail.com

\section{Introdução}

O abandono precoce da psicoterapia é um fenômeno significativo, frequentemente encontrado por terapeutas em diversas abordagens teóricas, com taxas variando entre $15 \%$ a $75 \%$ (Arnow et al., 2007; Bados, Balaguer, \& Saldanã, 2007). Entre os fatores associados ao abandono, destacam-se fatores do relacionamento terapeuta-paciente, especialmente a aliança terapêutica (AT). A metanálise de Sharf, Primavera e Diener (2010) revelou uma relação relativamente forte entre AT e abandono (d: 0,55), indicando que pacientes com ATs mais fracas têm mais chances de abandonar precocemente o tratamento. $\mathrm{O}$ estudo de moderadores sugere que a relação entre AT e abandono é mais forte em indivíduos com menor escolaridade, terapias mais longas e no contexto de internação. Estabelecer e manter uma boa aliança é, portanto, decisivo para evitar o abandono do tratamento e criar as condições para que o progresso terapêutico possa ocorrer (Horvath, Del Re, Flückiger, \& Symonds, 2011; Krause, Altimir \& Horvath, 2011).

O conceito de AT tem origem na consideração de Freud sobre a necessidade do paciente se ligar positivamente ao terapeuta para que o trabalho analítico possa se desenvolver. Os termos aliança terapêutica e aliança de trabalho foram cunhados, respectivamente por Zetzel e Greenson, que indicaram seu caráter consciente, não conflitivo e diferenciado da transferência (Gomes, 2015). Contudo, o conceito evoluiu, passando a ser concebido panteoricamente, a partir da concepção de Bordin (1979), como uma relação de colaboração mútua, consciente e proposital, entre paciente e terapeuta na terapia, caracterizada por um acordo sobre objetivos, uma atribuição de tarefas (combinadas no contrato terapêutico), e o desenvolvimento de laços afetivos. Essa visão 
da AT, panteórica e com ênfase na colaboração e no consenso, influenciou, ainda que de modo diverso e não consensual, a maior parte das principais medidas atuais do construto (Horvath et al., 2011; Krause et al., 2011).

AT hoje é reconhecida como um processo dinâmico que apresenta variações na intensidade, frequência e duração, dependendo do diagnóstico do paciente e do tipo de abordagem teórica empregada. Essas oscilações, denominadas de rupturas, podem acontecer ao longo do tratamento e, inclusive, ao longo de uma mesma sessão (Barros, Altimir \& Pérez, 2016; Safran, Muran, \& Eubanks, 2011). As rupturas são caracterizadas por uma deterioração na AT, manifestada pela falta de colaboração em tarefas ou objetivos ou uma tensão no vínculo emocional e na dificuldade em negociar aspectos da relação terapêutica (Eubanks, Muran, \& Safran, 2015; Safran \& Muran, 2006; Safran, Muran, \& Proskurov, 2009). O paciente pode evitar o trabalho terapêutico e/ou o terapeuta ou confrontá-los diretamente. Esses movimentos de rupturas da AT são inevitáveis em qualquer psicoterapia (Eubanks et al., 2015). Porém, quando não reparadas, as rupturas podem ocasionar o abandono do tratamento (Safran et al., 2011).

Existe ampla evidência de que as alianças enfraquecidas estão correlacionadas com o término unilateral do paciente (Doran, 2016; Safran et al., 2009; Safran, Israel, \& Einstein, 2006), bem como interações negativas (isto é, hostis e agressivas) entre paciente e terapeuta estão associadas a desfechos desfavoráveis (Coady, 1991; Samstag et al., 2008; Zilcha-Mano, Muran, Eubanks, Safran, \& Winston, 2018). Conforme a metanálise de Safran et al. (2011) existem evidências empíricas de que indivíduos com transtornos de personalidade apresentam mais intensidade de rupturas no início da terapia do que aqueles sem distúrbios de personalidade. Entre os primeiros, os pacientes com Transtornos de Personalidade Borderline (TPB) são os que apresentam uma taxa de rupturas maior. Possivelmente isso explique as maiores taxas de abandono de tratamento entre pacientes com este transtorno (Kröger, Harbeck, Armbrust, \& Kliem, 2013; Koons et al., 2001; Linehan et al., 2007; McMain et al., 2009).

Pacientes com TPB apresentam padrões inflexíveis e duradouros de dificuldades emocionais e interpessoais (Benjamin, 1993; Leichsenring, Leibing, Kruse, New, \& Leweke, 2011) que exigem do terapeuta o manejo diferenciado de aspectos centrais de sua patologia (Barnow et al., 2009; Kernberg, 2012; Lazarus, Cheavens, Festa, \& Zachary Rosenthal, 2014; Skodol et al., 2002). Por exemplo, a instabilidade afetiva e falta de integração do self e dos outros significativos é manifestada através de um sentimento de vazio crônico, percepções de si mesmo e do outro contraditórias e empobrecidas, dificultam a experiência empática e a relação com o terapeuta. Por este motivo, recomenda-se que as intervenções com esses pacientes focalizem o desenvolvimento e a manutenção da AT (Bennett, Parry, \& Ryle, 2006; Geremia, Benetti, Esswein, \& Bittencourt, 2016).

Como a aliança é tão crítica para o resultado, Safran e colaboradores (Eubanks, Muran, \& Safran, 2014; Muran et al., 2009; Safran \& Muran, 1996, 2000; Safran et al., 2011; Safran et al., 2009; Safran et al., 2006; Safran, Muran, \& Samstag, 1994) criaram e testaram um modelo para reparar rupturas na aliança durante a terapia. Recentemente, com o objetivo de sistematizar a codificação de rupturas e reparações da AT, Eubanks et al. (2015) desenvolveram o Rupture Resolution Rating System (3RS). O sistema visa a codificação das rupturas da AT e intervenções de reparações, em segmentos de sessões de psicoterapia.

Gersh et al. (2017) realizaram uma pesquisa com 44 jovens com TPB, visando explorar os processos de ruptura e reparação da AT, por meio do $3 \mathrm{RS}$. O estudo mostrou que as rupturas ocorreram em 53\% das sessões e com o passar do tratamento tendem a aumentar, sendo as de confronto mais frequentes. As rupturas que ocorreram no início do tratamento foram associadas com piores resultados. Por outro lado, uma maior resolução das rupturas foi associada a melhores resultados e podem ser oportunidades de crescimento terapêutico.

Considerando a relevância do processo da AT para a adesão de pacientes com TPB em psicoterapia, esse estudo tem como objetivo identificar a frequência e as variações dos processos 
de ruptura da AT em um caso interrompido de psicoterapia psicanalítica, realizada com paciente com diagnóstico de TPB e objetiva, ainda, descrever as características do processo terapêutico global.

\section{Método}

Estudo de caso sistemático, idiográfico, longitudinal e intensivo. Este tipo de estudo tem semelhanças com os estudos de caso da tradição clínica, mas se diferencia destes, entre outros aspectos, por apresentar maior rigor metodológico, como o uso de juízes independentes, analise de sessões gravadas em áudio e vídeo, e controle sobre vieses do pesquisador (Edwards, 2007; Serralta, Nunes, \& Eizirik, 2011).

\section{O processo em estudo e seus participantes}

$\mathrm{O}$ caso analisado consiste no processo de uma psicoterapia psicanalítica. O tratamento foi interrompido pelo paciente na $15^{\mathrm{a}}$ sessão. O paciente (denominado ficticiamente Carlos) possuía 30 anos. As suas queixas iniciais foram a falta de controle emocional e a dificuldade nos relacionamentos interpessoais e com sua parceira amorosa. Carlos foi atendido em um consultório particular de psicologia. A terapeuta era do sexo feminino, possuía 32 anos e formação em psicoterapia psicanalítica. O diagnóstico de TPB foi realizado pela terapeuta, com base na sua experiência clínica e na aplicação da Shedler-Westen Assessment Procedure - SWAP-200 (Shedler \& Westen, 1998; Westen \& Shedler, 1999), um instrumento do tipo Q-sort formado por 200 afirmativas que descrevem aspectos cognitivos, afetivos, relacionais, de pacientes com problemas de personalidade. Para a interpretação do perfil, utiliza-se escores padronizados. Escores T > 60 são compatíveis com o diagnóstico categórico de transtorno de personalidade, enquanto escores T $>55$ indicam a presença de traços destes. Conforme dados obtidos pela SWAP-200, Carlos apresentava características compatíveis com o diagnóstico de Transtorno de Personalidade Borderline, e características histriônicas. Os traços patológicos mais proeminentes eram a desregulação emocional e a psicopatia. Seu índice de saúde psicológica era indicativo de um nível médio de patologia da personalidade.

\section{Instrumentos}

Ficha de acompanhamento do processo. Esta ficha foi elaborada pela pesquisadora para ser preenchida pela terapeuta. Trata-se de um registro das sessões agendadas, a frequência do paciente na sessão, contemplando eventuais atrasos, e intercorrências dignas de nota (como por exemplo, falha no equipamento de gravação).

O Rupture Resolution Rating System (3RS) (Eubanks et al., 2015) é um sistema de observação de rupturas na AT para obtenção de uma classificação do tipo de ruptura (evitação ou confrontação) em uma sessão de psicoterapia, bem como as estratégias de resolução do terapeuta. Essa classificação é feita em uma escala de significância de 5 pontos, sendo 1 (sem significância) e 5 (alta significância). Na sequência, o avaliador atribui uma classificação de qual ruptura predominou na sessão, com base na frequência. O último item de avaliação refere-se a extensão em que o terapeuta causou ou exacerbou rupturas na sessão. O sistema apresenta alta confiabilidade entre avaliadores (Eubanks, Lubitz, Muran, \& Safran, 2018a). A versão em português do manual 3RS foi desenvolvida pela equipe.

O Psychotherapy Process Q-Set (PQS) (Jones, 2000) é um instrumento panteórico, do tipo Q-sort que apresenta 100 itens que descrevem as atitudes e vivências do paciente, as ações e atitudes do terapeuta e a natureza da interação entre ambos. Ao observar uma sessão terapêutica, o avaliador classifica os itens numa escala de 9 pontos na qual, de um lado, classificam-se as características identificadas como mais proeminentes do processo terapêutico (positivamente salientes) e em outro, as menos características (negativamente salientes). Os itens colocados nas 
categorias centrais são considerados neutros ou irrelevantes. A distribuição forçada segue a curva normal e evita o efeito halocêntrico. O ordenamento é, geralmente, realizado por dois ou mais juízes treinados. A versão em Português do Brasil do PQS foi desenvolvida por Serralta et al. (2007), e apresentou equivalência semântica com o instrumento original em inglês e coeficientes de fidedignidade entre avaliadores previamente treinados comparáveis com os obtidos com o instrumento original (Correlações Intraclasse superiores a 0,70).

\section{Procedimentos}

Essa pesquisa foi aprovada pelo Comitê de Ética em Pesquisa da Universidade do Vale do Rio dos Sinos (CAAE 39120214.6.0000.5344). As 15 sessões de psicoterapia deste caso foram gravadas em áudio e transcritas na íntegra para posterior análise por meio do 3RS. A quantificação das rupturas foi realizada nos segmentos de cinco minutos em todas as sessões por duplas de juízes. Foram 6 juízes: dois estudantes de Mestrado, um estudante de Doutorado, uma Psicóloga Doutora em Psicologia Clínica (todos psicoterapeutas de orientação psicanalítica), e dois estudantes de iniciação científica, sem experiência clínica. Os juízes receberam um treinamento de 20 horas, conforme orientação do manual do 3RS (Eubanks et al., 2015). Neste estudo, os juízes obtiveram um grau de concordância substancial $(\mathrm{K}=0,760 ; \mathrm{z}=8,0001 ; p>0,0001)$ na identificação de rupturas.

Para identificação das rupturas, o juiz deve estar atento durante a exibição do vídeo aos indicadores de diminuição da colaboração entre paciente e terapeuta, discordâncias dos objetivos e tarefas do tratamento, levando em conta os aspectos verbais e não-verbais do paciente. $\mathrm{O}$ processo de codificação das rupturas é complexo e envolve muitas etapas e foi descrito detalhadamente por Dotta (2019).

As análises feitas com o 3RS incluíram a frequência das rupturas ao longo do tratamento e a frequência da contribuição da terapeuta para as rupturas. Após, avaliou-se a média da ocorrência das rupturas tanto de evitação como de confrontação, a média do impacto específico das subcategorias das rupturas da AT, e em cada sessão, e o impacto global das rupturas na AT em todas as sessões de psicoterapia.

Para a análise do processo com o PQS, cada sessão foi codificada por duplas de juízes previamente treinados, formadas por codificadores diferentes daqueles que avaliaram as rupturas com o 3RS. Os avaliadores do PQS foram: 1 doutora em psicologia e 2 estudantes de doutorado, com experiência clínica e 1 estudante de graduação em Psicologia, bolsista de Iniciação Científica, sem experiência clínica. Os juízes eram cegos quanto ao número de sessões, resultado do tratamento e identidade de avaliações de outros juízes. Os juízes apresentaram boa fidedignidade no julgamento das sessões analisadas, com coeficiente de correlação Intraclasse entre 0,71 e 0,86. Para obter o resultado da descrição global do processo terapêutico, calculou-se e ordenou-se a média dos 10 itens do PQS mais e menos característicos do processo.

\section{Resultados}

\section{Processo global da psicoterapia}

A terapia durou 27 semanas. Contudo, devido às faltas frequentes entre as sessões, o paciente compareceu somente a 15 delas. Em quase a metade das sessões $(46,7 \%)$, o paciente chegou atrasado. A duração média das 15 sessões foi de 34,25 minutos $(D P=11,3)$. Entre a $1^{\mathrm{a}}$ e $4^{\mathrm{a}}$ sessão, observou-se uma média de duração de 29, 25 minutos $(D P=6,07)$. Entre a $5^{\mathrm{a}}$ e $11^{\mathrm{a}}$ sessão o tempo de duração da psicoterapia aumentou, durando em média 4,87 minutos $(D P=8,98)$. A sessão de número 7 teve a sua gravação interrompida devido à falta de bateria na câmera.

$\mathrm{O}$ processo terapêutico global avaliado com o PQS mostrou que a terapia apresentou poucos silêncios (item 12) e foi permeada por material significativo (item 88). A dupla discutiu temáticas relacionadas à situação de vida atual do paciente (item 69), bem como suas aspirações 
(item 41), geralmente apresentando um foco específico (item 23) na discussão dos relacionamentos interpessoais (item 63) e amorosos (item 64) do paciente. Durante as sessões, o paciente sentia-se seguro e confiante (item 44), era ativo (item 15) e iniciava os assuntos (item 25). Geralmente mostrava aceitar os comentários da terapeuta (item 42) e possuía facilidade para compreender os comentários da terapeuta (item 5).

A terapeuta mostrou-se autoconfiante (item 86) e comunicou-se por meio de um estilo claro e coerente (item 46). Na relação com Carlos, foi responsiva, envolvida (item 9) e mostrou possuir tato (item 77). Suas intervenções visaram obter mais informações e elaboração (item 31) e facilitar a fala do paciente (item 3), mantendo atenção a aspectos verbais, sem referir aos não-verbais.

A identificação das rupturas, na figura 4 ilustra o número de marcadores de ruptura identificados em cada sessão do tratamento. Identificou-se rupturas em todas as sessões de psicoterapia. Ao longo das 15 sessões, foram encontrados 100 marcadores de rupturas (em média, 6,66 rupturas por sessão). Destes, $69 \%$ são rupturas de evitação e $31 \%$, rupturas de confrontação.

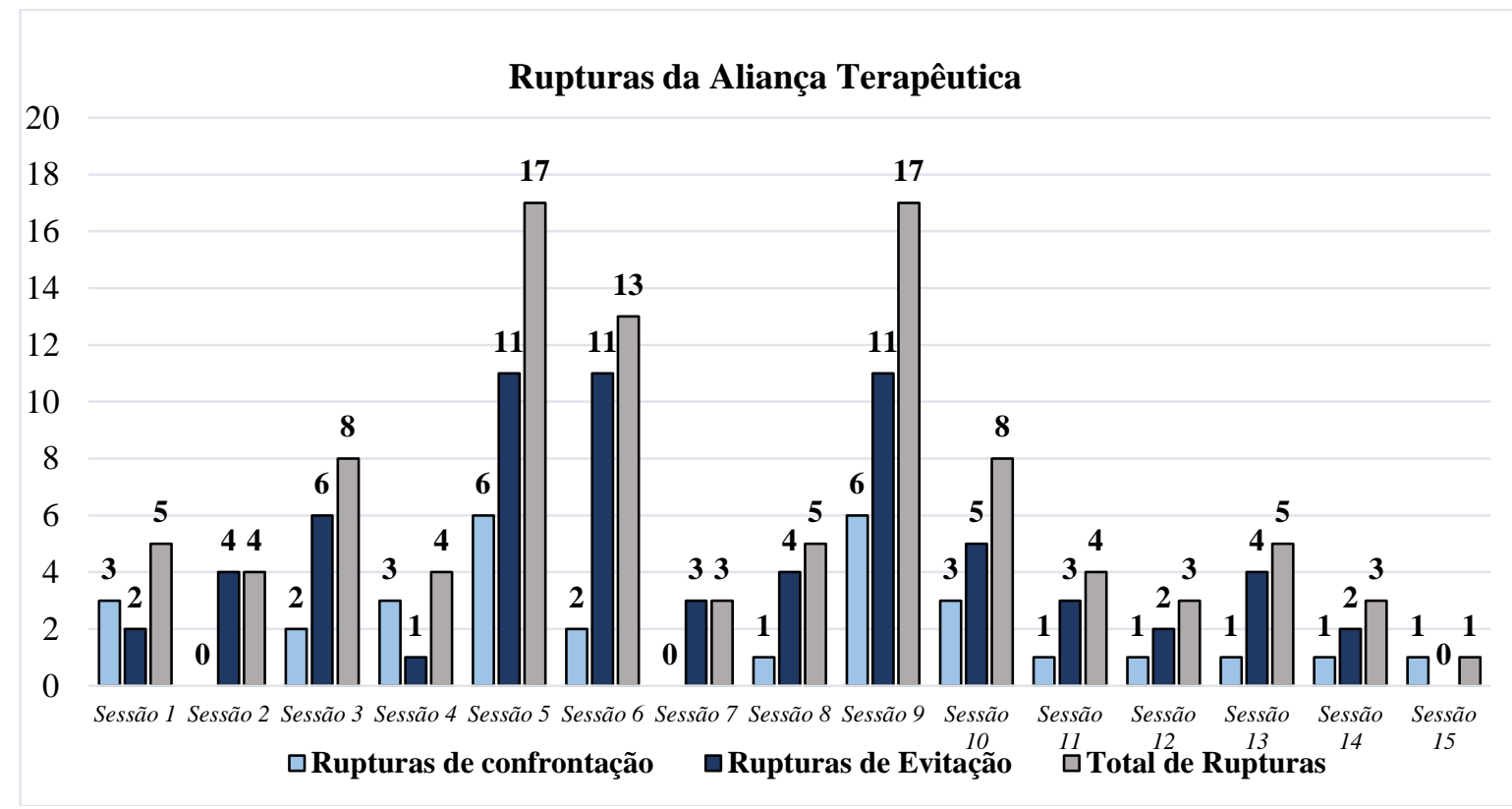

Figura 1. Número de marcadores de ruptura identificados.

Conforme a figura 1 , as sessões com maior ocorrência de rupturas foram a 5 , a 6 e a 9 (com 17, 13 e 17 rupturas, respectivamente). Já as com menor ocorrência, foram a 7, 12 e 14 com 3 rupturas cada, e a sessão15, com apenas uma. Observa-se uma distribuição de frequência bimodal, na qual identificou-se dois principais picos de aumento de rupturas (sessão 5 e 9). Quanto ao tipo de ruptura, com exceção da $1^{\mathrm{a}}$, da $4^{\mathrm{a}}$ e da última sessões, houve predominância de rupturas de evitação em todas as sessões.

As rupturas de evitação com maior frequência média ao longo do tratamento foram: mudança de tópico/narrativa de evitação $(\mathrm{Fr}=11)$, deferente e apaziguador $(\mathrm{Fr}=9)$, e comunicação abstrata $(\mathrm{Fr}=6)$. Já as rupturas de confrontação predominantes foram: paciente rejeita a intervenção do terapeuta $(\mathrm{Fr}=12)$, defende-se contra o terapeuta $(\mathrm{Fr}=3)$ e faz esforços para controlar o terapeuta $(\mathrm{Fr}=3)$. As demais frequências das rupturas podem ser verificadas na tabela 1 . 
Tabela 1

Ocorrência médias de marcadores das subcategorias das rupturas identificados nas 15 sessões do tratamento

\begin{tabular}{|c|c|c|c|c|c|c|}
\hline \multirow{7}{*}{ 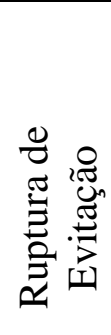 } & & $\mathrm{Fr}$ & Mín & Máx & $\mathrm{M}$ & DP \\
\hline & Negação & 5 & 1 & 2 & 1,20 & 0,44 \\
\hline & Resposta mínima & 0 & - & - & - & - \\
\hline & Comunicação abstrata & 6 & 1 & 2 & 1,50 & 0,54 \\
\hline & Mudança de Tópico/Narrativa de Evitação & 11 & 1 & 5 & 2,36 & 1,12 \\
\hline & Deferente e apaziguador & 9 & 1 & 6 & 2,44 & 1,87 \\
\hline & Conteúdo/afeto cindido & 6 & 1 & 1 & 1,00 & 0,00 \\
\hline \multirow{8}{*}{ 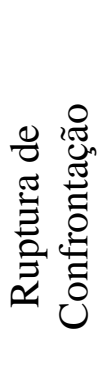 } & Autocrítica e desesperança & 0 & - & - & - & - \\
\hline & Queixas sobre o terapeuta & 0 & - & - & - & - \\
\hline & Paciente rejeita as intervenções do terapeuta & 12 & 1 & 4 & 1,50 & 0,90 \\
\hline & Queixas sobre as atividades da terapia & 0 & - & - & - & - \\
\hline & Queixas sobre os parâmetros da terapia & 1 & 1 & 1 & 1,00 & - \\
\hline & Queixas sobre o progresso da terapia & 0 & - & - & - & - \\
\hline & Paciente defende-se contra o terapeuta & 3 & 1 & 4 & 2,33 & 1,52 \\
\hline & Esforços para controlar o terapeuta & 3 & 1 & 2 & 1,67 & 0,57 \\
\hline
\end{tabular}

Foi examinada a intensidade do impacto das rupturas de confrontação e de evitação. A intensidade das rupturas de confrontação $(M=3,61 ; D P=0,9)$ foi ligeiramente maior quando comparada com o impacto das rupturas de evitação $(M=2,97 ; D P=1,1)$. As rupturas de confrontação de maior intensidade foram: paciente defende-se contra o terapeuta $(M=3,67 ; D P=$ $0,5)$; esforços para controlar e pressionar o terapeuta $(M=3,67 ; D P=1,12)$ e paciente rejeita as intervenções do terapeuta $(M=3,11 ; D P=1,0)$. A ruptura queixas sobre os parâmetros da terapia $(M=4,00)$ apresentou apenas uma ocorrência, e, portanto, o grau de impacto 4 refere-se somente a esse marcador. Entre as rupturas de evitação, os marcadores com maior impacto foram negação $(M=3,33 ; D P=1,4)$; comunicação abstrata $(M=3,17 ; D P=1,0)$ e deferente e apaziguador $(M=$ $3,09 ; D P=0,9)$. As demais médias dos impactos das subcategorias específicas das rupturas de evitação e confrontação podem ser visualizadas na tabela 2.

Tabela 2

Impacto médio de cada subcategoria das rupturas da AT ao longo das 15 sessões do tratamento

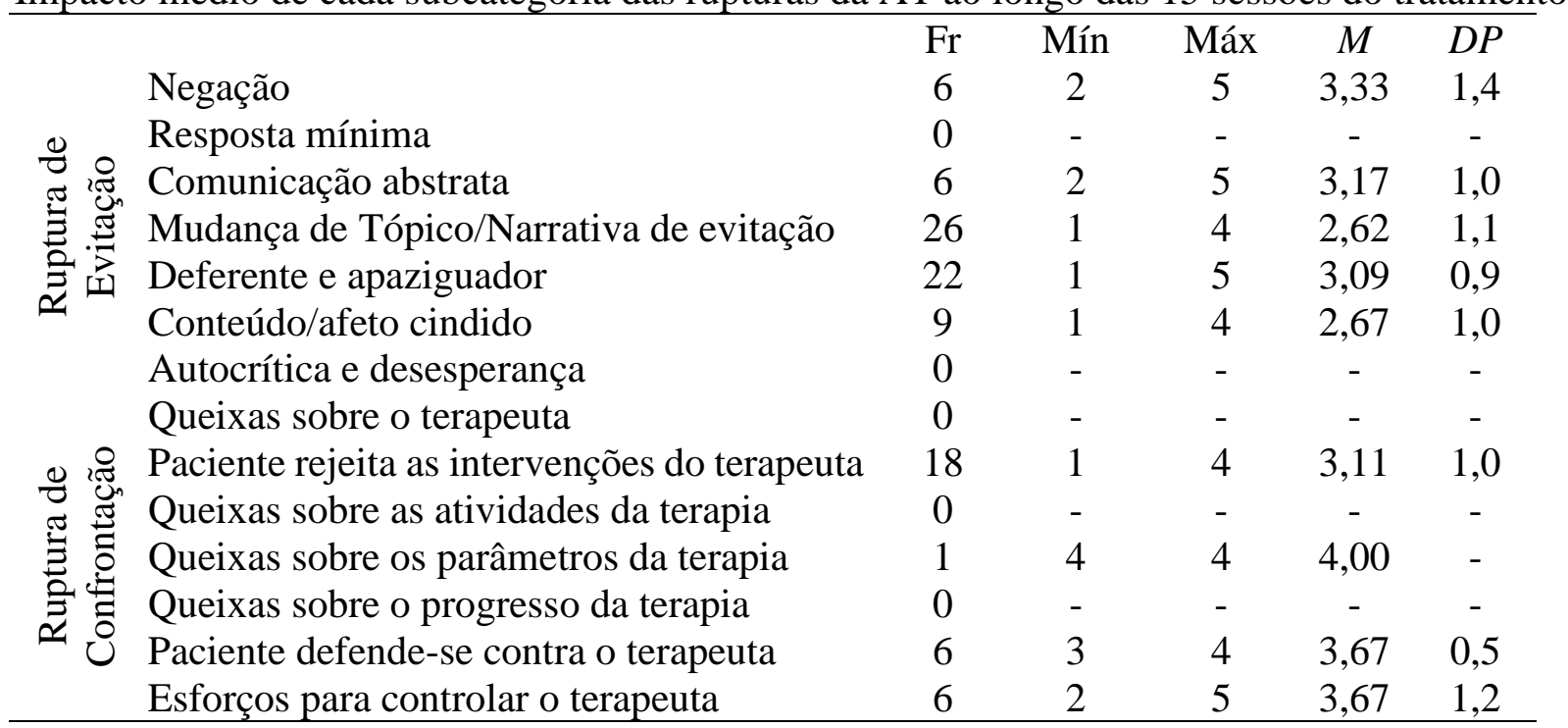


Constatou-se que as rupturas (de confrontação e evitação) apresentaram, em média, alguma significância sobre a AT $(M=3,0 ; D P=0,62)$. Em todas as sessões, as rupturas tiveram ao menos algum impacto na AT (pontuação de pelo menos 3,0). Nas sessões 5, 6, 9, 13 e 14 houve presença de rupturas com alto impacto (pontuação 5,0). As sessões que apresentaram, em média, maior impacto na AT foram a $12(M=3,7 ; D P=1,10), 13(M=4,2 ; D P=1,10)$ e $14(M=4,0 ; D P=1,10)$.

Ao longo do tratamento, identificou-se 30 contribuições da terapeuta no sentido de provocar ou exacerbar as rupturas. As sessões em que a terapeuta contribuiu com maior frequência para a ocorrência das rupturas foram a $5(n=10), 6(n=6), 8$ e $9(n=5$ em cada), embora essa conduta também tenha sido observada nas sessões 7, 10 e 11. Em contraposição, não houve contribuições da terapeuta para as rupturas nas sessões 1, 2, 3, 4, 12, 13, 14 e 15.

\section{Discussão}

A psicoterapia de Carlos foi compreendida como uma terapia malsucedida, pois o paciente abandonou o tratamento unilateralmente e sem que os ganhos terapêuticos pretendidos tenham sido alcançados. O tratamento teve uma duração de 27 semanas. Contudo, apesar da frequência contratada semanal, totalizou somente 15 sessões.

Além disto, denotou-se o baixo número de sessões em relação ao tempo de tratamento deveu-se, sobretudo às faltas entre sessões (foram 9 faltas; destas, somente uma combinada com a terapeuta). Nota-se que as faltas iniciaram na $4^{\mathrm{a}}$ sessão, justamente quando, segundo a literatura, espera-se que a AT comece a se desenvolver de modo mais efetivo (Gersh et al., 2017).

Quando se examina o sequenciamento de presenças e faltas nas sessões, chama à atenção que, com exceção das sessões iniciais, o processo é praticamente todo intercalado por faltas. Isso possivelmente reflete a dificuldade da dupla de desenvolver uma AT suficientemente boa no início do tratamento (Horvath et al., 2011). Como o paciente buscou espontaneamente a psicoterapia, expressando necessidade de ajuda e, aparentemente, concordou com as combinações iniciais do contrato, há um sinal claro de de desacordo sobre as tarefas da terapia (Bordin, 1979; Krause, Altimir \& Horvath, 2011) que se manifesta nas ausências e atrasos frequentes.

Modelos efetivos de tratamento para TPB compartilham, entre outros aspectos, foco na experiência emocional, uma maior atividade do terapeuta, e foco no relacionamento terapêutico (Weinberg, Ronningstam, Goldblatt, Schechter, \& Maltsberger, 2011). A descrição global do processo com o PQS não captura nenhum desses elementos, embora existam itens do instrumento que descrevam tais características. E ainda, esta descrição evidencia uma "aparente colaboração" na exploração de material significativo nas sessões, relacionados aos problemas que levaram o paciente ao tratamento. É significativa também a ausência de discussão de comportamentos não verbais que podem vincular-se a resistências e dificuldades na interação.

Deve-se atentar para o fato de que a análise global com o PQS levar em conta o macroprocesso em psicoterapia e centrar-se na observação ampla de como paciente e terapeuta vivenciam o processo terapêutico. Assim como uma fotografia, captura-se uma cena como um todo e se obtém uma visão panorâmica do processo, com baixo grau de resolução (Bucci, 2007; Cordioli \& Grevet, 2018).Assim, para melhor compreender o processo da AT, por meio do 3RS pretendeu-se atentar para questões específicas, detalhadas e imediatas de como paciente e terapeuta organizam-se durante a sessão de psicoterapia (o microprocesso), observando a cena terapêutica com maior resolução para identificar os possíveis problemas com a AT (Barcellos, Cardon, \& Kieling, 2018; Bucci, 2007).

A avalição da AT analisada em um nível microscópico, ao longo do tratamento, permitiu identificar problemas na colaboração entre paciente e terapeuta no presente caso, já constatados em nível macro pelas faltas e atrasos mencionados. Identificou-se rupturas em todas as sessões, número elevado de rupturas $(n=100)$, em comparação com outros estudos realizados com o $3 R S$ com pacientes com TPB (Gersh et al., 2017). As rupturas foram predominantemente de evitação, 
mais frequentes nas sessões intermediárias (com a contribuição da terapeuta) e tiveram algum impacto na AT. E ainda, foi observado que após a elevação das rupturas, nas sessões intermediárias, houve decréscimo da frequência. Porém, há um aumento do seu impacto na AT, nas sessões que antecederam o término e nas quais a terapeuta contribuiu para as rupturas.

A diferença entre as frequências das rupturas do presente estudo, quando comparadas com alguns relatos na literatura, é um achado interessante e inesperado. Contudo, o padrão é consistente com os demais indicadores evidenciados (faltas e atrasos). Não se pode também desconsiderar que se trata de uma psicoterapia interrompida (malsucedida) com paciente TPB, supostamente mais propenso a demonstrarem dificuldades no estabelecimento e manutenção da AT (Boritz, Barnhart, Eubanks, \& McMain, 2018; Safran et al., 2009). O estudo de Doran, Safran e Muran (2017), que examinou a relação entre o processo terapêutico e a negociação da AT de 47 pacientes com diferentes psicopatologias clínicas (ansiedade e depressão) e com transtorno de personalidade (48,9\%), também destacou flutuação na ocorrência das rupturas (confrontação e evitação) ao longo do tempo.

Bennett et al. (2006) apontam que maior ocorrência de rupturas da AT e abandono do tratamento são comuns em pacientes com TPB. Estes pacientes apresentam falhas no processo de representação do self e outros (Caligor, Kernberg, \& Clarkin, 2008) e sob ativação emocional, tendem ao pensamento concreto, não mentalizado (Bateman, Campbell, Luyten, \& Fonagy, 2018), apresentando comportamentos impulsivos, relacionados às falhas na regulação emocional, que levam a uma rápida distorção sobre si mesmos e da relação com seus terapeutas (Caligor et al., 2008; Leichsenring et al., 2011). Essa distorção sobre a relação com o terapeuta favorece a ocorrência e o impacto das rupturas na AT (Spinhoven, Giesen-Bloo, van Dyck, Kooiman, \& Arntz, 2007).

Durante o tratamento, Carlos utilizava rupturas de evitação em maior frequência: mudança de tópico/narrativa de evitação, deferência e apaziguamento, mudança de tópico e comunicação abstrata. Tal achado sugere que o paciente apresente dificuldade de expressar sua insatisfação ou angústia, de maneira direta. Conforme a literatura, a frequência de rupturas de evitação é significativamente maior em pacientes que abandonaram o tratamento (Boritz et al., 2018). Tais rupturas, em comparação com as de confrontação, são mais difíceis de identificar, pois o comportamento do paciente, ao evitar o trabalho terapêutico ou a terapeuta é sutil, pouco claro ou até mesmo obscurecido (Safran et al., 2011). As rupturas de evitação podem estar disfarçadas de uma conformidade ou engajamento superficial, por exemplo (Boritz et al., 2018).

O comportamento de evitação pode então ser visto como uma estratégia para regular a emoção intensa e avassaladora que é ativada no contexto da relação terapêutica (Bernecker, Levy, \& Ellison, 2014). Nesse sentido, o estudo realizado por Cash, Hardy, Kellett \& Parry (2014) aponta que a resolução da ruptura não ocorria quando os terapeutas discutiam ou exploravam diretamente as rupturas, mas sim quando eles abordavam a ruptura indiretamente mudando sua abordagem para explorar questões que eram mais importantes para o paciente. No presente estudo, contudo, as estratégias de reparação das rupturas não foram analisadas especificamente. Não obstante, os resultados sugerem que na ausência de pistas explícitas das rupturas de evitação, a terapeuta pode ter se posicionado de forma mais passiva diante das mesmas, tendo dificuldade para identificá-las e/ou repará-las.

As contribuições da terapeuta na ocorrência das rupturas sugerem que a terapeuta parecia ignorar os marcadores de ruptura, e não estar ciente do que estava acontecendo no nível da relação, subestimando o impacto destas quebras da colaboração terapêutica no processo. Um estudo indica que a contribuição do terapeuta para as rupturas é preditivo de abandono do tratamento (Eubanks et al., 2018b). Além disso, a literatura destaca um aparente paradoxo no trabalho de reparação das rupturas com pacientes TPB: lidar com as rupturas diretamente pode ser considerado excessivo e ameaçador para alguns indivíduos, embora não abordá-la diretamente possa exacerbar ainda mais a ruptura (Boritz et al., 2018). 
Apesar da predominância das rupturas de evitação, as de confrontação merecem atenção. Diante delas, o paciente, maioritariamente, rejeitou as intervenções da terapeuta, defendeu-se dela e se esforçou para controlar o processo terapêutico ou a terapeuta. Rupturas de confrontação ocorreram em 13 das 15 sessões do tratamento. Os juízes notaram ainda que, ao rejeitar as intervenções da sua terapeuta, o paciente demostrava um tom de deboche, fazia uso de comentários sarcásticos e descrevia comportamentos agressivos. Um estudo feito por Gülüm, Soygüt e Safran, (2018) identificou o humor sarcástico como uma terceira categoria de ruptura. O sarcasmo é uma forma sutil de comunicação agressiva, que expressa a dificuldade em verbalizar sentimentos sobre a terapia ou terapeuta, levando à desistência do tratamento, nas sessões posteriores.

$\mathrm{Na}$ fase final da psicoterapia, a diminuição das rupturas está associada à resolução destas (Eubanks et al., 2018b). Em contraste, no presente caso, as últimas sessões não constituíram fase final, pois numa terapia supostamente de longo prazo o processo idealmente estaria em uma etapa intermediária (Luz, 2015). Desse modo, a diminuição da ocorrência de rupturas observada nas últimas sessões (sessões 12, 13 e 14), associada ao aumento da sua significância em termos do impacto na AT, parece denunciar uma deterioração da AT que pode ter levado à desistência do paciente em relação à terapia.

É digno de nota que as sessões em que as rupturas tiveram maior impacto na AT tenham sido as que antecederam a última sessão, quando o paciente fala da sua decisão de interromper o tratamento. Vale destacar ainda que essas sessões foram as que menos apresentaram rupturas, e que nelas não se observaram rupturas que mostrassem expressões de autocrítica e desesperança, queixas sobre a terapeuta, sobre atividades da terapia ou progresso do tratamento. Constata-se, portanto, que inúmeras rupturas com menor importância podem anteceder rupturas com maior significância. A hipótese a ser verificada é de que a não resolução de rupturas menos significativas leve a um incremento da piora da AT, culminando com o abandono.

As rupturas também podem ser compreendidas como enactments, conceito que destaca o papel do inconsciente na relação entre paciente e terapeuta (Safran \& Kraus, 2014; Safran \& Muran, 2000). Para Safran \& Kraus (2014), as rupturas da AT são essencialmente movimentos inconscientes entre a dupla. $\mathrm{O}$ terapeuta fica involuntariamente envolvido no funcionamento do paciente, reencenando, assim, uma forma de relacionamento disfuncional que é característico do paciente. Tais processos, quando não se tornam conscientes e alvos do trabalho terapêutico, obstruem o desenvolvimento de um bom processo terapêutico. Por outro lado, a consciência do terapeuta sobre as flutuações contínuas na qualidade na AT pode apresentar oportunidades valiosas para mobilizar o processo de mudança no paciente.

A fase inicial ou de abertura do tratamento pode ser mais longa na terapia com pacientes com TPB, tendo em vista a necessidade de exploração das rupturas que podem interferir no estabelecimento e manutenção da AT. Além disso, torna-se importante a necessidade do cultivo da AT como o principal objetivo no tratamento, a adaptação da psicoterapia às características do paciente e o constante monitoramento sobre as oscilações da AT. A postura flexível e empática do terapeuta é essencial para lidar com as rupturas, tanto com as de evitação como de confrontação.

Destaca-se a necessidade de haver prudência na interpretação dos dados apresentados, tendo em vista as limitações deste estudo, de caráter exploratório. A codificação das rupturas da AT foi realizada pelo manual 3RS, que não apresenta categorias para avaliação e codificação das rupturas provocadas pelo terapeuta. A avaliação da medida de microprocesso envolveu somente a perspectiva do paciente. Embora os juízes tenham recebido treinamento e obtido concordância substancial, não se sabe até que ponto a codificação é consistente com a codificação do 3RS padrão ouro.

No item "contribuição do terapeuta para as rupturas" foi apenas identificada a frequência em que a terapeuta exacerbou ou contribuiu para elas. A avaliação das reparações da AT, que não foi alvo deste estudo, poderia esclarecer melhor o comportamento oscilatório da AT, ao longo do tratamento. 
Em estudos futuros sugere-se a inclusão da codificação das estratégias que os terapeutas podem usar para identificar e resolver efetivamente as rupturas da AT. Além disso sugere-se o exame das rupturas e reparações da AT em casos múltiplos, com diferentes psicopatologias e desfechos, bem como estudos que avaliem as diferenças na natureza e no valor preditivo das rupturas precoces e posteriores.

\section{Conclusão}

Esse estudo ofereceu hipóteses empiricamente sustentadas sobre o abandono precoce da terapia por parte de um paciente borderline. Ele revelou um processo permeado de muitas rupturas desde o início, as quais foram aumentando, primeiramente em número e após, em intensidade, culminando na sessão em que o paciente comunica o abandono. A terapeuta contribuiu para a ocorrência ou aumento das rupturas. Suas ações e intervenções apresentavam como foco os problemas do paciente, mas não da relação terapêutica. Em conjunto, estes fatores parecem ter tido relação com o abandono. No entanto, considerando as limitações do delineamento meramente descritivo e da ausência de análise específica no nível microprocessual das estratégias de resolução das rupturas, essa hipótese não foi testada.

É digno de nota que no Brasil, até o presente momento, não há avaliações empíricas das rupturas da AT. O sistema 3RS foi recentemente traduzido para o Português do Brasil pela equipe do Laboratório de Pesquisa em Psicoterapia e Psicopatologia (LAEPSI). Neste sentido, este é um estudo pioneiro no contexto nacional para o exame microprocessual da AT, constituindo um estudo exploratório que secundariamente visa introduzir estudos sobre a temática.

Não obstante, o estudo apresenta contribuições para a clínica ao trazer para discussão e análise o papel do relacionamento terapêutico no processo de mudança e no desfecho da psicoterapia. A teoria psicanalítica contemporânea enfatiza a influência mútua entre terapeuta e paciente e valoriza a autenticidade, a flexibilidade e a espontaneidade do terapeuta. A AT neste contexto, adquire um papel central e não apenas de suporte para a etapa inicial do tratamento. Com pacientes com transtornos da personalidade, como os borderline, que tipicamente apresentam dificuldades no desenvolvimento da aliança, o foco nesta dificuldade parece ser fundamental ao processo. Trabalhar as rupturas da aliança (isto é, antecipá-las, identificá-las e repará-las) pode proporcionar aos pacientes oportunidades de aprender a negociar a tensão entre as suas próprias dificuldades e a necessidade de ajuda versus as dificuldades e as necessidades do relacionamento terapêutico, de uma maneira nova e mais construtiva.

Dada a evidência empírica indicando que a AT é um ingrediente crítico de mudança em diversas formas de terapia, parece importante desenvolver um corpo de pesquisa bem articulado e, de conhecimento relevante sobre medidas microprocessuais da AT. Este estudo é o primeiro desenvolvido no país a utilizar uma abordagem baseada em observadores externos para avaliação microprocessual das rupturas da AT, e a explorar como esses processos se desdobram ao longo do tempo na terapia. Outros estudos deverão contribuir futuramente para a ampliação desse tipo de investigação no país. Investigações nacionais e internacionais de outros casos individuais, bem como estudos de casos múltiplos e comparativos sobre estes microprocessos, desenvolvido junto a pacientes com distintos diagnósticos, desfechos e abordagens terapêuticas poderão futuramente trazer mais luz sobre a complexa questão da contribuição do relacionamento terapêutico e da aliança para a adesão, o abandono, o sucesso e/ou o insucesso da psicoterapia.

\section{Referências}

Arnow, B. A., Blasey, C., Manber, R., Constantino, M. J., Markowitz, J. C., Klein, D. N., ... Rush, A. J. (2007). Dropouts versus completers among chronically depressed outpatients. Journal of Affective Disorders, 97(1-3), 197-202. doi: 10.1016/j.jad.2006.06.017 
Bados, A., Balaguer, G., \& Saldaña, C. (2007). The Efficacy of Cognitive-Behavioral Therapy and the Problem of Drop-Out. Journal of Clinical Psychology, 63(6), 585-592. doi: 10.1002/jclp

Bateman, A., Campbell, C., Luyten, P., \& Fonagy, P. (2018). A mentalization-based approach to common factors in the treatment of borderline personality disorder. Current Opinion in Psychology, 21, 44-49. doi: 10.1016/j.copsyc.2017.09.005

Barros, P., Altimir, C., \& Pérez, J. C. (2016). Patients' facial-affective regulation during episodes of rupture of the therapeutic alliance/Regulación afectivo-facial de pacientes durante episodios de ruptura de la alianza terapéutica. Estudios de Psicología, 37(2-3), 580-603. doi: 10.1080/02109395.2016.1204781

Barcellos, M., Cardon, L., \& Kieling, C. (2018). Evidências em Psicoterapia. Em A. . Cordioli \& E. H. Grevet (Eds.), Psicoterapia. Abordagens Atuais ( $4^{\text {th }}$ ed.). Porto Alegre: Artmed.

Barnow, S., Stopsack, M., Grabe, H. J., Meinke, C., Spitzer, C., Kronmüller, K., \& Sieswerda, S. (2009). Interpersonal evaluation bias in borderline personality disorder. Behaviour Research and Therapy, 47(5), 359-365. doi: 10.1016/j.brat.2009.02.003

Benjamin, L. . (1993). Diagnosis and treatment of personality disorders: A structural approach. New York: G. Press, Ed.

Bennett, D., Parry, G., \& Ryle, A. (2006). Resolving threats to the therapeutic alliance in cognitive analytic therapy of borderline personality disorder: A task analysis. Psychology and Psychotherapy: Theory, Research and Practice, 79(3), 395-418. doi: $10.1348 / 147608305 X 58355$

Bernecker, S. L., Levy, K. N., \& Ellison, W. D. (2014). A meta-analysis of the relation between patient adult attachment style and the working alliance. Psychotherapy Research, 24(1), 1224. doi: 10.1080/10503307.2013.809561

Bordin, E.S. (1979). The generalizability of the psychoanalytic concept of the working alliance. Psychoterapy: Theory, Research and Practice, 16(3), 252-260.

Boritz, T., Barnhart, R., Eubanks, C. F., \& McMain, S. (2018). Alliance Rupture and Resolution in Dialectical Behavior Therapy for Borderline Personality Disorder. Journal of Personality Disorders, 32(Supplement), 115-128. doi: 10.1521/pedi.2018.32.supp.115

Bucci, W. (2007). Pesquisa sobre processo. Em E. Person, A. Cooper, \& G. Gabbard (Eds.), Compêndio de Psicanálise (1 ${ }^{\text {st }}$ ed.), (pp. 320-336). Porto Alegre: Artmed.

Caligor, E., Kernberg, O. F., \& Clarkin, J. (2008). Psicoterapia dinâmicas das patologias leves da personalidade. Porto Alegre: Artmed.

Cash, S. K., Hardy, G. E., Kellett, S., \& Parry, G. (2014). Alliance ruptures and resolution during cognitive behaviour therapy with patients with borderline personality disorder. Psychotherapy Research, 24(2), 132-145. doi: 10.1080/10503307.2013.838652

Coady, N. (1991). Therapist Interpersonal Processes and Outcomes in Psychodynamic Psychotherapy. Reasearch on Social Work Practice, 1(2), 122-138.

Cordioli, A. V., \& Grevet, E. H. (2018). Psicoterapias: Abordagens Atuais (4 ${ }^{\text {th }}$ ed.). Porto Alegre: Artmed.

Doran, J. M. (2016). The working alliance: Where have we been, where are we going? Psychotherapy Research, 26(2), 146-163. doi: 10.1080/10503307.2014.954153

Doran, J. M., Safran, J. D., \& Muran, J. C. (2017). An Investigation of the Relationship Between the Alliance Negotiation Scale and Psychotherapy Process and Outcome. Journal of Clinical Psychology, 73(4), 449-465. doi: 10.1002/jclp.22340

Dotta, P. (2019). Rupturas da Aliança Terapêutica: ilustração de um sistema de avaliação e análise de um caso de abandono em psicoterapia. Dissertação de Mestrado, Universidade do Vale do Rio dos Sinos - UNISINOS, Programa de Pós-graduação em Psicologia Clínica, Rio Grande do Sul, São Leopoldo, Brasil.

Edwards, D. J. (2007). Collaborative Versus Adversarial Stances in Scientific Discourse: 
Implications for the Role of Systematic Case Studies in the Development of Evidence-Based Practice in Psychotherapy. Pragmatic Case Studies in Psychotherapy, 3(1), 6-34. doi: 10.14713/pcsp.v3i1.892

Eubanks, C. F., Muran, J. C., \& Safran, J. D. (2014). Alliance-focused training. Psychotherapy Theory Research Practice Training, 52(2), 169-173. doi: 10.1037/a0037596

Eubanks, C. F., Muran, J. C., \& Safran, J. D. (2015). Rupture Resolution Rating System (3RS): Manual. ResearchGate, (January), 1-16. doi: 10.13140/2.1.1666.8488

Eubanks, C. F., Lubitz, J., Muran, J. C., \& Safran, J. D. (2018a). Rupture Resolution Rating System (3RS): Development and validation. Psychotherapy Research, (January), 1-16. doi: 10.13140/2.1.1666.8488

Eubanks, C. F., Sinai, M., Israel, B., Muran, J. C., \& Safran, J. D. (2018b). Alliance Rupture Repair: A Meta-Analysis. Psychotherapy, 55(4), 508-519. doi: 10.1037/pst0000185.supp

Geremia, L., Benetti, S. P. C., Esswein, G. C., \& Bittencourt, A. A. (2016). A aliança terapêutica no paciente diagnosticado com transtorno de personalidade borderline. Perspectivas Em Psicologia, 20(2). doi: 10.14393/PPv20n2a2016-03

Gersh, E., Hulbert, C. A., McKechnie, B., Ramadan, R., Worotniuk, T., \& Chanen, A. M. (2017). Alliance rupture and repair processes and therapeutic change in youth with borderline personality disorder. Psychology and Psychotherapy: Theory, Research and Practice, 90(1), 84-104. doi: 10.1111/papt.12097

Gomes, F. G. (2015). Aliança terapêutica e a relação real com o terapeuta. Em S. EIZIRIK, Cláudio L; Aguiar, Rogério W; Schestatsky (Ed.), Psicoterapia de orientação analítica : fundamentos teóricos e clínicos (3 ed.) (pp. 238-248). Porto Alegre: Artmed.

Gülüm, İ. V, Soygüt, G., \& Safran, J. D. (2018). A comparison of pre-dropout and temporary rupture sessions in psychotherapy. Psychotherapy Research, 28(5), 685-707. doi: 10.1080/10503307.2016.1246765

Horvath, A. O., Del Re, A. C., Flückiger, C., \& Symonds, D. (2011). Alliance in individual psychotherapy. Psychotherapy, 48(1), 9-16. doi: 10.1037/a0022186

Jones, E. E. (2000). Therapeutic Action: A guide to psychoanalytic therapy. New Jersey: Aronson. Kernberg, O. (2012). The Inseparable Nature of Love and Aggression: Clinical and Theoretical Perspectives. Washington: American Psychiatric Association Publishing.

Koons, C. K., Robins, C. J., Tweed, J. L., Lynch, T. R., Gonzalez, A. M., Mogs, J. Q., ... Bastian, L. A. (2001). Efficacy of Dialectical Behavior Therapy in Women Veterans With Borderline Personality Disorder. Behavior Therapy, 32, 371-390. doi: 10.1016/S0005-7894(01)80009-5

Krause, M., Altimir, C., \& Horvath, A. (2011). Deconstructing the therapeutic alliance: Reflections on the underlying dimensions of the concept. Clinical and Health, 22(3), 267283. doi: $10.5093 / \mathrm{cl} 2011 \mathrm{v} 22 \mathrm{n} 3 \mathrm{a} 7$

Kröger, C., Harbeck, S., Armbrust, M., \& Kliem, S. (2013). Effectiveness, response, and dropout of dialectical behavior therapy for borderline personality disorder in an inpatient setting. Behaviour Research and Therapy, 51(8), 411-416. doi: 10.1016/j.brat.2013.04.008

Lazarus, S. A., Cheavens, J. S., Festa, F., \& Zachary Rosenthal, M. (2014). Interpersonal functioning in borderline personality disorder: A systematic review of behavioral and laboratory-based assessments. Clinical Psychology Review, 34(3), 193-205. doi: 10.1016/j.cpr.2014.01.007

Leichsenring, F., Leibing, E., Kruse, J., New, A. S., \& Leweke, F. (2011). Borderline personality disorder. The Lancet, 377, 453-461.

Linehan, M. M., Comtois, K. A., Murray, A. M., Brown, M. Z., Gallop, R. J., Heard, H. L., ... \& Lindenboim, N. (2006). Two-year randomized controlled trial and follow-up of dialectical behavior therapy vs therapy by experts for suicidal behaviors and borderline personality disorder. Archives of general psychiatry, 63(7), 757-766. doi: 10.1001/archpsyc.63.7.757.

Luz, A. B. (2015). Fases da Psicoterapia. Em C. L. Eizirik, R. W. de Aguiar, \& S. S. Schestatsky 
(Eds.), Psicoterapia de Orientação Analítica: fundamentos teóricos e clínicos (pp. 249-266). Porto Alegre: Artmed.

McMain, S. F., Links, P. S., Gnam, W. H., Guimond, T., Cardish, R. J., Korman, L., \& Streiner, D. L. (2009). A randomized trial of dialectical behavior therapy versus general psychiatric management for borderline personality disorder. American Journal of Psychiatry, 166(December), 1365-1374. doi: 10.1176/appi.ajp.2009.09010039

Muran, J. C., Safran, J., Gorman, B. S., Samstag, L. W., Eubanks C.F., \& Winston, A. (2009). The relationship of early alliance ruptures and their resolution to process and outcome in three time-limited psychotherapies for personality disorders. Psychotherapy: Theory, Research, Practice, Training, 46(2), 233-248. doi: 10.1037/a0016085

Safran, J. D., Israel, B., \& Einstein, A. (2006). Has the concept of the therapeutic alliance outlived its usefulness ?, 43(3), 286-291. doi: 10.1037/0033-3204.43.3.286

Safran, J. D., \& Kraus, J. (2014). Alliance ruptures, impasses, and enactments: A relational perspective. Psychotherapy, 51(3), 381. doi: 10.1037/a0036815

Safran, J. D, \& Muran, J. C. (1996). The resolution of ruptures in the therapeutic alliance. Journal of Consulting and Clinical Psychology, 64(3), 447-458. doi: 10.1037/0022-006X.64.3.447

Safran, J. D., \& Muran, J. C. (2000). Resolving therapeutic alliance ruptures: Diversity and integration. Journal of Clinical Psychology, 56(2), 233-243. doi: 10.1002/(SICI)10974679(200002)56:2<233::AID-JCLP9>3.0.CO;2-3

Safran, J. D, \& Muran, J. C. (2006). Has the concept of the therapeutic alliance outlived its usefulness? Psychotherapy: Theory, Research, Practice, Training, 43(3), 286-291. doi: 10.1037/0033-3204.43.3.286

Safran, J. D, Muran, J. C., \& Eubanks, C.F. (2011). Repairing alliance ruptures. Psychotherapy, 48(1), 80-87. doi: 10.1037/a0022140

Safran, J. D., Muran, J. C., \& Proskurov, B. (2009). Alliance, negotiation, and rupture resolution. In Levy, R. A. Ablo. J. S. Gabbard, G. O. Handbook of evidence-based psychodynamic psychotherapy (pp. 201-225). Humana Press.

Safran, J. D, Muran, J. C., \& Samstag, L. W. (1994). Resolving therapeutic alliance ruptures: A task analytic investigation. The Working Alliance: Theory, Research, and Practice, (July 2015), 225-255.

Samstag L. W., Muran, J. C., Wachtel, P. L., Slade, A., Safran, J. D., \& Winston M. D, A. (2008). Evaluating Negative Process: A Comparison of Working Alliance, Interpersonal Behavior, and Narrative Coherency Among Three Psychotherapy Outcome Conditions. American Journal of Psychotherapy, 62(2), 165-194.

Sharf, J., Primavera, L. H., \& Diener, M. J. (2010). Dropout and therapeutic alliance: A metaanalysis of adult individual psychotherapy. Psychotherapy: Theory, Research, Practice, Training, 47(4), 637-645. doi: 10.1037/a0021175

Serralta, F. B., Nunes, M. L. T., \& Eizirik, C. L. (2007). Elaboração da versão em português do Psychotherapy Process Q-Set. Revista de Psiquiatria Do Rio Grande Do Sul, 29(1), 44-55. doi: 10.1590/S0101-81082007000100011

Serralta, F B, Nunes, M. L. T., \& Eizirik, C. L. (2011). Considerações metodológicas sobre o estudo de caso na pesquisa em psicoterapia. Estudos de Psicologia (Campinas), 28(4), 501510.

Shedler, J., \& Westen, D. (1998). Refining the Measurement of Axis II: A Q-Sort Procedure for Assessing Personality Pathology. Assessment, 5(4), 333-353. https://doi.org/10.1177/107319119800500403

Skodol, A. E., Gunderson, J. G., Pfohl, B., Widiger, T. A., Livesley, W. J., \& Siever, L. J. (2002). The borderline diagnosis I: psychopathology, comorbidity, and personaltity structure. Biological Psychiatry, 51(12), 936-950. doi: 10.1016/S0006-3223(02)01324-0

Spinhoven, P., Giesen-Bloo, J., van Dyck, R., Kooiman, K., \& Arntz, A. (2007). The therapeutic 
alliance in schema-focused therapy and transference-focused psychotherapy for borderline personality disorder. Journal of Consulting and Clinical Psychology, 75(1), 104-115. doi: 10.1037/0022-006X.75.1.104

Weinberg, I., Ronningstam, E., Goldblatt, M. J., Schechter, M., \& Maltsberger, J. T. (2011). Common factors in empirically supported treatments of borderline personality disorder. Current psychiatry reports, 13(1), 60-68. doi: 10.1007/s11920-010-0167-x

Westen, D., \& Shedler, J. (1999). Revising and Assessing Axis II, Part I: Developing a Clinically and Empirically Valid Assessment Method. American Journal of Psychiatry, (2), 258-272. doi: 10.1016/j.desal.2014.06.029

Zilcha-Mano, S., Muran, J. C., Eubanks, C. F., Safran, J. D., \& Winston, A. (2018). When therapist estimations of the process of treatment can predict patients rating on outcome: The case of the working alliance. Journal of Consulting and Clinical Psychology, 86(4), 398-402. doi: $10.1037 / \mathrm{ccp} 0000293$

Financiamento: Este estudo foi financiado pelo Conselho Nacional de Desenvolvimento Científico e Tecnológico (CNPq) - Edital Universal ( $n^{\circ}$ 28/2008) e apoiado pela Coordenação de Aperfeiçoamento de Pessoal de Nível Superior - Brasil (CAPES) - Código 001.

Participação dos autores: a) Planejamento e concepção do trabalho; b) Coleta de dados; c) Análise e interpretação de dados; d) Redação do manuscrito; e) Revisão crítica do manuscrito.

P.D.L. contribuiu em a,b,c,d,e; .L.P.F. em b,c,d,e; F.B.S. em a,b,c,d,e.

Editora científica responsável: Dra. Cecilia Cracco 